WH1 (Invited)

8:30 am - 9:00 am

\title{
Optical Clocks and Frequency Synthesis Using Femtosecond Lasers ${ }^{\star}$
}

\author{
Scott A. Diddams \\ Time and Frequency Division \\ National Institute of Standards and Technology \\ 325 Broadway, Mail Stop 847 \\ Boulder, CO 80305 USA \\ Phone: (303) 497-7459, FAX: (303) 497-7845 \\ Email: sdiddams@boulder.nist.gov
}

\begin{abstract}
This paper describes recent advances in the design and control of femtosecond laser combs for their use in optical clocks and in the synthesis of low noise microwave and optical signals.
\end{abstract}

\section{Introduction}

Since mode-locked femtosecond lasers were introduced into the field of optical frequency metrology about four years ago they have become indispensable tools in this exciting and expanding area of research [1]. It is now widely accepted that femtosecond lasers will play a critical role in the next generation of atomic clocks based on optical frequencies [2,3]. Moreover, in the near future it is likely that stable optical oscillators (i.e. lasers) will be one of the sources with the lowest phase noise in any region of the electromagnetic spectrum. The modelocked femtosecond laser can function as a synthesizer to transfer the low-noise properties of such optical oscillators to the microwave domain, or to virtually any visible or near-infrared frequency. In this talk, we will describe the important properties and control mechanisms of femtosecond lasers for use in optical clocks and low noise frequency synthesis. Recent results and known limitations will be presented. In addition, the role of femtosecond lasers in searches for possible time-variations of fundamental constants [4], and the coherent control of ultrafast pulses $[5,6]$ will be presented.

\section{Femtosecond laser-based synthesizers}

A femtosecond laser and its associated frequency comb can be viewed as a general purpose, extremely broadband synthesizer with either an optical or microwave frequency reference (Fig. 1). The output of the synthesizer can similarly be an optical or microwave frequency. To accomplish this, it is required that both degrees of freedom of the associated frequency comb be measured and controlled. The connection between optical and microwave domains is understood most readily in the frequency domain, where the spectrum of the femtosecond laser consists of a comb of evenly-spaced modes with frequencies given by

$f_{\mathrm{n}}=n f_{\mathrm{r}}+f_{0}$

Here, $f_{\mathrm{r}}$ is the repetition frequency of the laser (typically 0.1 to $1 \mathrm{GHz}$ ), $n$ is an integer, and $f_{0}$ is a common offset frequency---called the carrier-envelope offset frequency---due to dispersion in the laser cavity.

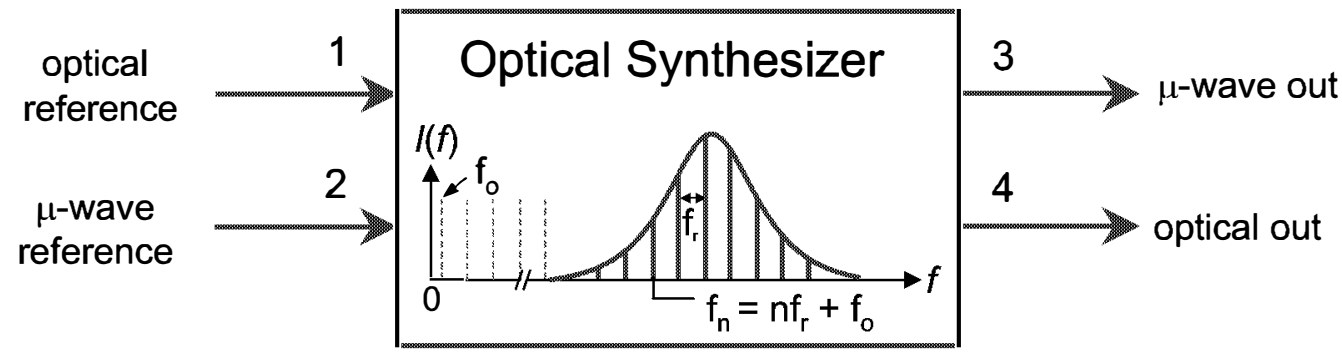

Figure 1: The frequency domain output of a femtosecond laser is an array of discrete frequencies separated by the laser's repetition frequency $f_{\mathrm{r}}$. The entire comb is offset by the frequency $f_{0}$. Measuring and controlling both $f_{\mathrm{r}}$ and $f_{0}$ provides a connection between these two microwave frequencies and the optical frequency $f_{\mathrm{n}}$. As such, the femtosecond laser can be viewed as a general purpose, extremely broadband synthesizer with optical and microwave inputs and outputs. 
In recent experiments we have tested the performance of the femtosecond laser synthesizer for optical-tooptical synthesis (ports $1 \rightarrow 4$ ) and optical-to-microwave synthesis (ports $1 \rightarrow 3$ ). In the case of optical-tooptical synthesis, the validity of Eq. (1) has been verified at the $10^{-19}$ level by comparing multiple femtosecond laser synthesizers that employ broadband Ti:sapphire lasers [7] as well as narrowband lasers with additional spectral broadening in nonlinear microstructure fiber [8]. These results appear to be limited mainly by noise of a technical nature (thermal and mechanical fluctuations) and total integration time. Additionally, Hertz-level linewidths have been achieved for all the comb elements when the femtosecond laser is referenced to a stabilized CW laser oscillator [9]. In the case of optical-to-microwave synthesis, we have demonstrated the generation of $10 \mathrm{GHz}$ signals down converted from the optical domain with unprecedented phase noise (-100 $\mathrm{dBc} / \mathrm{Hz}$ at $1 \mathrm{~Hz}$ offset $)$ and fractional frequency instability $\left(3 \times 10^{-15}\right.$ at $1 \mathrm{~s}$ of averaging). Such extremely lownoise microwave signals should be useful for applications in radar, optical analog-to-digital conversion, and other experiments requiring precise timing.

\section{Acknowledgement}

The author would like to acknowledge and thank the following people for their contributions to this work: Leo Hollberg, Albrecht Bartels, Chris Oates, Guido Wilpers, Nathan Newbury, Jim Bergquist, Windell Oskay, Long-Sheng Ma, Bi Zhiyi, Lennart Robertsson, Massimo Zucco, and Robert Windeler.

* Contribution of an agency of the U.S. govemment; not subject to copyright in the U.S.

\section{References}

[1]. Th. Udem, R. Holzwarth, and T. W. Hänsch, Nature. 416, 233 (2002).

[2]. S. A. Diddams, Th. Udem, J. C. Bergquist, E. A. Curtis, R. E. Drullinger, L. Hollberg, W. M. Itano, W. D. Lee, C. W. Oates, K. R. Vogel, D. J. Wineland, Science, 293, 825 (2001).

[3]. J. Ye, L.-S. Ma, J. L. Hall, Phys.Rev. Lett. 87, 270801/1-4 (2001).

[4] S. Bize, S. A. Diddams, U. Tanaka, C. E. Tanner, W. H. Oskay, R. E. Drullinger, T. E. Parker, T. P. Heavner, S. R. Jefferts, L. Hollberg, W. M. Itano, D. J. Wineland, J. C. Bergquist, Phys. Rev. Lett. 90, 150802 (2003).

[5]. R.K. Shelton, L.-S. Ma, H.C. Kapteyn, M.M. Murnane, J.L. Hall, and J. Ye, Science 293, 1286 (2001).

[6]. A. Bartels, N. R. Newbury, I. Thomann, L. Hollberg, S. A. Diddams, Opt Lett. 29, 403 (2004).

[7]. A. Bartels and H. Kurz, Opt. Lett. 27, 1839 (2002).

[8]. L.-S. Ma, Z. Bi, A. Bartels, L. Robertsson, M. Zucco, R. S. Windeler, G. Wilpers, C. Oates, L. Hollberg, S. A. Diddams, Science, 303, 1843 (2004).

[9]. A. Bartels, C.W. Oates, L. Hollberg, S.A. Diddams, Opt. Lett. 29, 1081 (2004). 